\title{
Preterm Infants Harbour a Rapidly Changing Mycobiota That Includes Candida Pathobionts
}

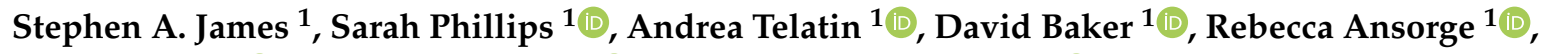 \\ Paul Clarke 2,3® , Lindsay J. Hall $1,4, *(\mathbb{D}$ and Simon R. Carding $1,3, * \mathbb{B}$ \\ 1 Gut Microbes and Health, Quadram Institute Bioscience, Norwich Research Park, Norwich NR4 7UQ, UK; \\ steve.james@quadram.ac.uk (S.A.J.); sarah.phillips@quadram.ac.uk (S.P.); \\ andrea.telatin@quadram.ac.uk (A.T.); david.baker@quadram.ac.uk (D.B.); \\ rebecca.ansorge@quadram.ac.uk (R.A.) \\ 2 Neonatal Intensive Care Unit, Norfolk and Norwich University Hospitals NHS Foundation Trust, \\ Norwich NR4 7UY, UK; paul.clarke@nnuh.nhs.uk \\ 3 Norwich Medical School, University of East Anglia, Norwich NR4 7TJ, UK \\ 4 Ziel-Institute for Food and Health, Technical University of Munich, 85354 Freising, Germany \\ * Correspondence: lindsay.hall@quadram.ac.uk (L.J.H.); simon.carding@quadram.ac.uk (S.R.C.)
}

Received: 26 October 2020; Accepted: 5 November 2020; Published: 9 November 2020

\begin{abstract}
Fungi and the mycobiome are a fundamental part of the human microbiome that contributes to human health and development. Despite this, relatively little is known about the mycobiome of the preterm infant gut. Here, we have characterised faecal fungal communities present in 11 premature infants born with differing degrees of prematurity and mapped how the mycobiome develops during early infancy. Using an ITS1 sequencing-based approach, the preterm infant gut mycobiome was found to be often dominated by a single species, typically a yeast. Candida was the most abundant genus, with the pathobionts C. albicans and C. parapsilosis highly prevalent and persistent in these infants. Gestational maturity at birth affected the distribution and abundance of these Candida, with hospital-associated C. parapsilosis more prevalent and abundant in infants born at less than 31 weeks. Fungal diversity was lowest at 6 months, but increased with age and change of diet, with food-associated Saccharomyces cerevisiae most abundant in infants post weaning. This study provides a first insight into the fungal communities present within the preterm infant gut, identifying distinctive features including the prominence of pathobiont species, and the influence age and environmental factors play in shaping the development of the mycobiome.
\end{abstract}

Keywords: mycobiome; GI tract; preterm infant; early life; pathobiont; fungi; yeast; Candida parapsilosis

\section{Introduction}

The human gastrointestinal (GI) tract harbours a complex microbial ecosystem comprising a vast number and array of bacteria, archaea, fungi, protozoa and viruses that constitute the intestinal microbiota [1,2]. Most of what we currently know regarding the contribution the enteric microbiota makes to human health and disease comes from the analysis of the prokaryome. Various studies of bacterial constituents of the microbiota have provided evidence for their ability to influence various physiological processes including digestion, micronutrient biosynthesis, energy homeostasis, pathogen colonisation resistance, and maintenance of epithelial barrier integrity [3], and the development and functional maturation of the host immune system [4]. Disruption of the prokaryome (dysbiosis) in early life can have a profound and long-lasting impact on health later in life, including the development of non-communicable illnesses, such as atopy and allergic disease (e.g., asthma), and metabolic disorders [5-8]. 
By comparison, far less is known about the mycobiome and fungal residents of the GI tract, and their involvement in human health and disease. This is in part related to their low abundance [9]. In a recent metagenomic study, fungi were estimated to make up approximately $0.1 \%$ of the microbiota in the healthy adult GI tract [1]. In considering that fungi are larger in volume than bacteria, they are likely to constitute a sizeable proportion of the intestinal microbiota biomass [10]. Thus, despite their low abundance, fungi can have significant impacts on human health [11]. The yeast Saccharomyces boulardii, a probiotic strain of $S$. cerevisiae, is frequently prescribed as an effective treatment for antibiotic-associated diarrhoea and irritable bowel syndrome (IBS) [12-15]. In contrast, fungal dysbiosis and reduced fungal diversity, coupled with increased levels of Candida parapsilosis and Pichia jadinii (C. utilis) has been observed in paediatric cases of inflammatory bowel disease (IBD) [16]. IBD pathogenesis has also been correlated with an increased Basidiomycota/Ascomycota ratio and increased abundance of C. albicans [17]. Fungal dysbiosis has also been reported in colorectal adenoma, and in metabolic syndrome and obesity [17-19]. Fungal pathobiont overgrowth leading to infection, often as a result of prolonged antibiotic exposure, also poses a significant health risk, especially in immune deficient individuals, such as those undergoing chemotherapy and solid organ transplantation [11].

Preterm infants represent a highly vulnerable and 'at risk' patient group, having endured disrupted fetal development, with the final weeks/months of development occurring ex utero in a neonatal intensive care unit (NICU). A shortened gestational period, shortened labour and possible delivery by caesarean section are all factors that can contribute to reduced exposure to maternal vaginal and enteric microbiotas [20,21]. Indeed, the bacterial composition of the preterm gut is distinct, characterised by a reduced diversity and dominance of facultative anaerobes (e.g., Enterococcus and Staphylococcus spp.) [22,23]. Preterm infants also have an underdeveloped immune system and an intestinal epithelial barrier with increased permeability. Consequently, this makes them highly susceptible to nosocomial infection, especially from the 'ESKAPE' pathogens, a group of virulent and multi-drug resistant bacteria which include Enterococcus faecium, Klebsiella pneumoniae and Staphylococcus aureus [24]. In addition, these infants are at increased risk of developing late-onset sepsis and necrotising enterocolitis [25-27]. Broad-spectrum antibiotics are also frequently administered to preterm infants in their first weeks of life. However, an adverse effect of such treatment is increased susceptibility to fungal infections resulting in significant morbidity and mortality [28]. These can arise either from overgrowth of resident fungal pathobionts (e.g., C. albicans) or from horizontally acquired species within the hospital environment (e.g., C. parapsilosis) [29-34]. To reduce the risk of such infections, prophylactic antifungals are routinely administered in the first weeks of life to the highest risk preterm babies, specifically those born at less than 27 weeks' gestation and/or weighing less than $750 \mathrm{~g}$, while they have an indwelling central venous catheter.

In the present study, we developed laboratory protocols and a fungal analysis bioinformatics pipeline to characterise the faecal mycobiome of a small cohort of young infants all born with differing degrees of prematurity, over the course of a 12-month period in early infancy. The goal was to gain a first insight into the composition and diversity of the preterm infant gut mycobiome, and how it changes between the first 6 to 18 months' postnatal life; an important developmental window in early infant life.

\section{Materials and Methods}

\subsection{Study Participants and Samples}

The infant cohort comprised 11 infants, 5 females and 6 males, aged less than 2-years old. All were born preterm at the Norfolk and Norwich University Hospital (NNUH), with gestational ages ranging from 25 weeks (extremely preterm) to 36 weeks (late preterm), with a median age of $30+4$ weeks. All 11 infants were participants in the BAMBI study (Reference no: '2012/2013—42HT'), a longitudinal microbiota profiling study to determine the impact of Bifidobacterium and Lactobacillus supplementation on the bacterial composition of the preterm infant gut [35]. All subjects gave their informed consent 
for inclusion before they participated in the study. The study was conducted in accordance with the Declaration of Helsinki, and the protocol was approved by the Quadram Institute Bioscience Ethics Committee and in accordance with protocols by the National Research Ethics Service (NRES) approved UEA/QIB Biorepository (License no: 11208). Clinical metadata for the infants including gender, gestational age at birth, birthweight, mode of delivery, and postnatal antimicrobial treatment, along with whether they received breastmilk and/or formula during their stay at the NNUH are provided in Table S1.

\subsection{DNA Extraction}

Infant faecal samples were collected at three time points; 6 months, 12 months and 18 months and were aliquoted and stored at $-70{ }^{\circ} \mathrm{C}$ prior to processing and DNA extraction. Total microbial DNA was extracted from $200 \mathrm{mg}$ of faeces using the FastDNA Spin Kit for Soil (MP Biomedicals, Irvine, CA, USA) and following the manufacturer's protocol. In addition, all samples were homogenized using a FastPrep-24 benchtop instrument (MP Biomedicals) at $6.0 \mathrm{~m} / \mathrm{s}$ for $3 \times 1 \mathrm{~min}$ with $5 \mathrm{~min}$ resting intervals on ice. Extracted DNA was quantified and quality checked using the Qubit 3.0 fluorometer and associated Qubit dsDNA BR Assay Kit (Thermo Fisher, Waltham, MA, USA), as well as DNA visualisation by stained agarose gel electrophoresis ( $1 \%$ agarose gel stained with Midori Green Direct DNA Stain). A flowchart outlining the DNA extraction protocol is shown in Figure S1 (left hand panel).

\subsection{ITS1 Amplification, Library Preparation and Sequencing}

The fungal ITS1 region was amplified from $100 \mathrm{ng}$ of faecal DNA by PCR using the ITS1F and ITS2 primer set [36,37], with each primer modified at the $5^{\prime}$ end to include an Illumina adapter tail using the following amplification conditions: $94{ }^{\circ} \mathrm{C}$ for $5 \mathrm{~min} ; 35$ cycles of $92{ }^{\circ} \mathrm{C}$ for $30 \mathrm{~s}, 55^{\circ} \mathrm{C}$ for $30 \mathrm{~s}$, and $72{ }^{\circ} \mathrm{C}$ for $45 \mathrm{~s}$; and a final extension of $72{ }^{\circ} \mathrm{C}$ for $5 \mathrm{~min}$. Amplification reactions were set up in duplicate for each faecal DNA sample, and positive and negative controls were also included in each PCR run (see Section 2.5). Following ITS1 PCR, a $0.7 \times$ SPRI purification using KAPA Pure Beads (Roche, Wilmington, MA, USA) was performed and the purified DNA was eluted in $20 \mu \mathrm{L}$ of EB buffer (10 $\mathrm{mM}$ Tris- $\mathrm{HCl})$. In a second PCR, library index primers were added using a Nextera XT Index Kit v2 (Illumina, Cambridge, UK) and following the following amplification conditions: $95^{\circ} \mathrm{C}$ for $5 \mathrm{~min}$ : 10 cycles of $95^{\circ} \mathrm{C}$ for $30 \mathrm{~s}, 55^{\circ} \mathrm{C}$ for $30 \mathrm{~s}$, and $72{ }^{\circ} \mathrm{C}$ for $30 \mathrm{~s}$; and a final extension of $72{ }^{\circ} \mathrm{C}$ for 5 min. Following PCR, libraries were quantified using the Invitrogen ${ }^{\mathrm{TM}}$ Quant-iT dsDNA high sensitivity assay kit (Thermo Fisher) and run on a FLUOstar Optima plate reader (BMG Labtech, Aylesbury, UK). Libraries were pooled following quantification in equal quantities. The final pool was SPRI cleaned using 0.7× KAPA Pure Beads, quantified on a Qubit 3.0 fluorometer and run on a High Sensitivity D1000 ScreenTape (Agilent Inc., Santa Clara, CA, USA) using the Agilent Tapestation 4200 to calculate the final library pool molarity. The pool was then run, at a final concentration of $8 \mathrm{pM}$, on an Illumina MiSeq instrument using the MiSeq ${ }^{\circledR}$ Nano v2 $(2 \times 250$ bp) Kit (Illumina). All sequencing was performed at the Quadram Institute Bioscience, Norwich. The raw data were analysed locally on the MiSeq instrument using MiSeq reporter.

\subsection{Mycobiome Characterisation}

Illumina MiSeq reads were analysed using a hybrid pipeline using USEARCH [38] and QIIME 2 [39]. After removal of locus-specific primers and reads with ambiguous bases using fastp 0.20.0 [40], the remaining reads were pooled, quality filtered and dereplicated using USEARCH. Identification of representative sequences was performed using the UNOISE3 algorithm [41], to produce a set of amplicon sequence variants (ASVs). A feature table was produced with USEARCH. Representative sequences and the feature table were imported as QIIME 2 artifacts. QIIME 2 was used to assign the taxonomy using the UNITE Fungal ITS database (release 04.02.2020) [42], to align ASVs using mafft [43] via "q2-alignment", and to construct a phylogeny with fasttree2 [44], using "q2-phylogeny". Community composition was assessed using R 3.6.2 [45] and the R package phyloseq [46]. The feature table, taxonomic classification, 
phylogeny and metadata were imported into R to create a phyloseq object. Every ASV with a zero count in all samples was removed to assess alpha diversity measures. Community composition and beta-diversity measures were assessed after removing samples with $<7500$ counts, transforming counts to relative abundances and removing taxa with $<5 \%$ relative abundances in all samples. A flowchart outlining the main steps in the mycobiome analysis pipeline is shown in Figure S1 (right hand panel)

The raw Illumina ITS1 sequence data produced by the present study have been deposited at the NCBI Short Reads Archive (SRA), under BioProject accession number PRJNA627184. All scripts used in this study are available at https:/github.com/quadram-institute-bioscience/bambi-its/.

\subsection{Inclusion of Controls}

Controls were included at each stage of this study. During DNA extraction, an empty bead-beating tube was included and treated exactly the same as for the tubes containing faecal samples, and was quantified and visualised similarly. This extraction control was included in the initial amplicon PCR

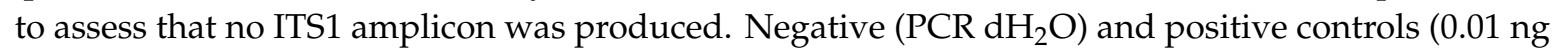
C. albicans DNA) were included for all PCR reactions. Libraries were also prepared from the DNA extraction control and from C. albicans DNA and were used as pipeline controls in the downstream bioinformatic analyses.

\section{Results}

\subsection{ITS1 Based Mycobiome Profiling and the Infant Gut Mycobiome}

We developed a culture-independent protocol and bioinformatics pipeline to amplify and sequence the fungal internal transcribed sequence (ITS) 1 region located between the $18 \mathrm{~S}$ and $5.8 \mathrm{~S}$ rRNA genes to gain a better insight into the composition and diversity of the infant gut mycobiome in early life, and how it changes over time. The protocol and pipeline was used to characterise the faecal fungal communities present in 11 healthy young infants comprising five females (F1 to F5) and six males (M1 to M6), all of whom were born premature (see Table S1). Faecal samples were collected over a 12-month period, at 6, 12 and 18 months. Following collection, samples were aliquoted and stored at $-70{ }^{\circ} \mathrm{C}$ until required. Although culture-dependent protocols were also employed in this study, prolonged sample freezing was found to prevent the isolation of viable fungi. A total of 29 faecal samples were obtained (for four infants only two samples were available) for ITS1 sequencing. A total of 1,609,183 quality-trimmed ITS1 reads were obtained, ranging from 8924 for infant F3, 18-month sample (F3_18) to 130,247 for M2_12, with a sample average of 55,489 reads (see Datasheet S1). One hundred and twelve fungal taxa were detected, with the majority $(86 \%)$ classified to the genus level or below. Two taxa could not be assigned at the phylum level or below, and were classified as 'Unidentified' (see Datasheet S1). The number of fungal taxa detected in each infant sample ranged from five to 37 , with a sample average of 15.8 (Table S2). A significant proportion of the fungi detected were infant and sample time point-specific $(47 / 112 ; 42.0 \%)$.

At the phylum level, $>98 \%$ of fungi belonged to either Ascomycota or Basidiomycota. Sixty taxa were ascomycetes, 44 of which could be identified to species level, while 50 taxa were basidiomycetes, and 34 were identified to species level (Table S3). Ascomycota was the predominant phylum, accounting for $60 \%$ of all ITS1 reads, and was dominant in many of the samples (Figure 1a). Ascomycetes were the most abundant fungi at all collection timepoints (Figure S2). 


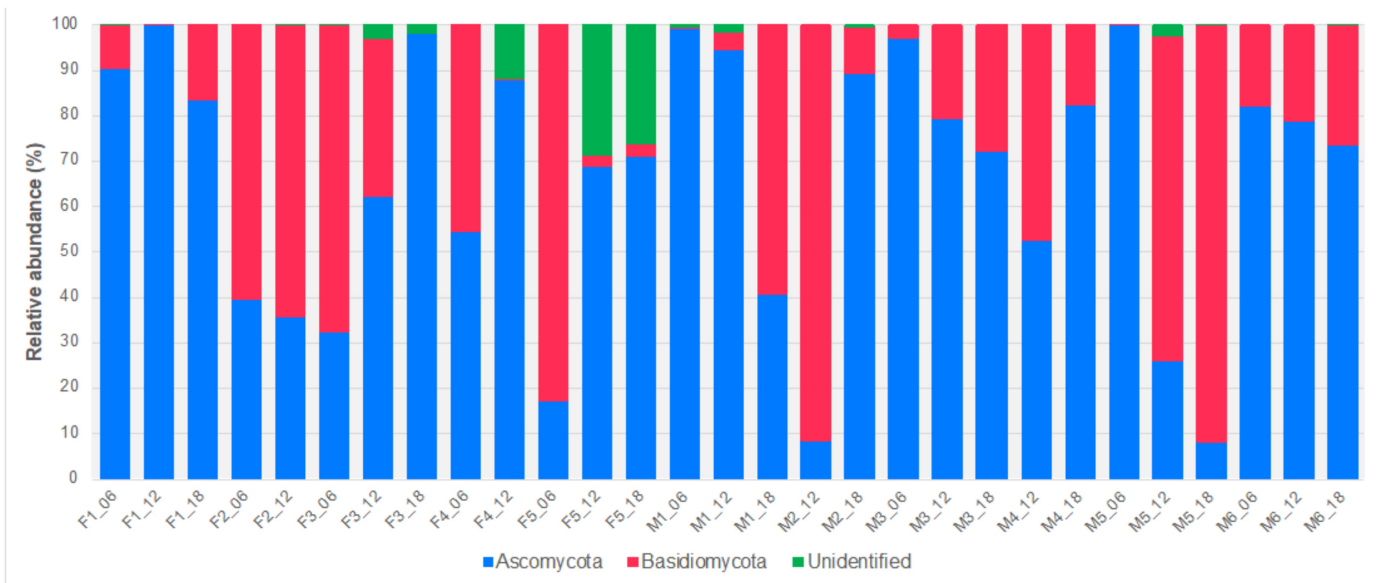

(a)

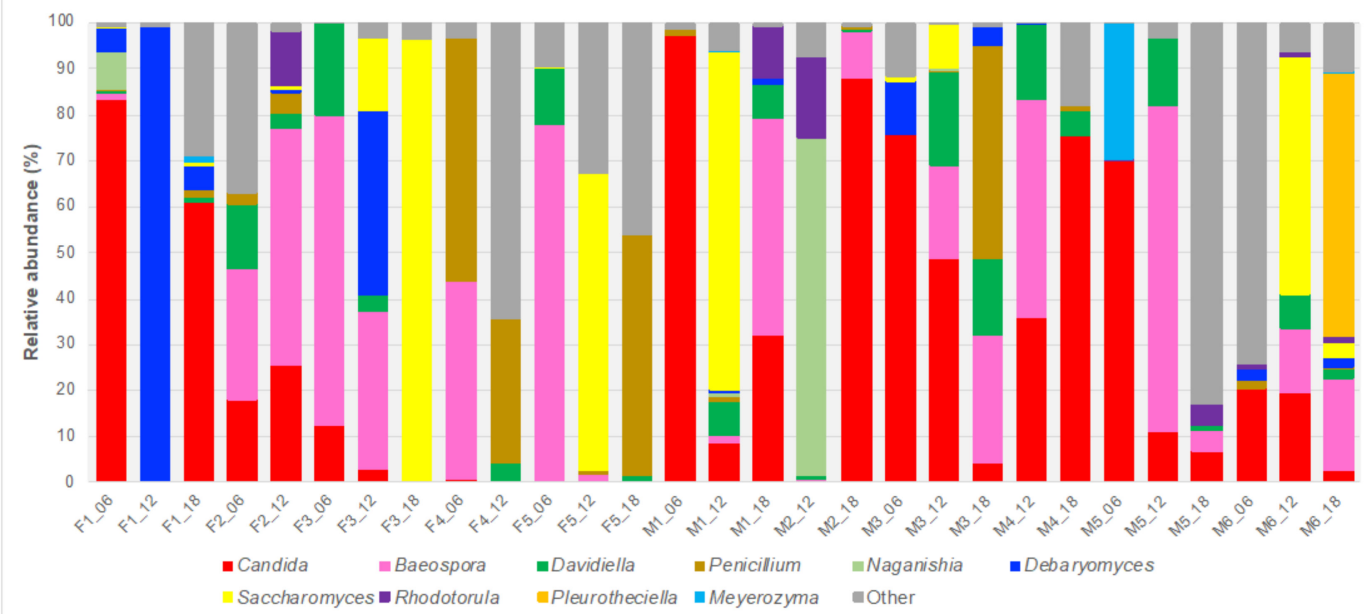

(b)

Figure 1. Bar charts of the relatively most abundant fungal taxa in the preterm infant gut at (a) phylum level and (b) genus level.

Candida was present in all 29 infant samples (Table S4). In some samples, such as the 6-month samples from infants F1, M1, M3 and M5, Candida was the predominant genus (Figure 1b). Other prevalent genera, of varying abundance, included Davidiella (23), Penicillium (21), Aspergillus (18), Saccharomyces (15) and Debaryomyces (14) (Table S4), some of which dominated individual samples (e.g., Debaryomyces, F1_12; Saccharomyces, F3_18) (Figure 1b). Amongst the Basidiomycota, Baeospora (e.g., B. myosura) was the most abundant and prevalent genus, present in all but two samples (Figure 1b; Table S4). Five other prevalent basidiomycetous genera were Malassezia (13), Rhodotorula (12), Filobasidium (9), Naganishia (8) and Vishniacozyma (8). However, all of these genera were present in low abundance, with individual mean abundances of 0.2 to $2.9 \%$ (Filobasidium and Naganishia, respectively) with many basidiomycetous genera occurring at abundances of $<0.2 \%$ (Table S4).

Among the ten most abundant genera of either phylum, six were yeast genera (i.e., Candida, Debaryomyces, Meyerozyma, Naganishia, Rhodotorula and Saccharomyces) (Figure 1b). Of the 78 fungi resolved to species level, six of the most abundant were yeasts. Moreover, these yeasts were subdivided into two distinct groups, human-associated (e.g., Candida albicans) and food-related (e.g., Saccharomyces cerevisiae) (Table S5).

\subsection{Prevalence of Opportunistic Candida Pathogens}

Candida accounted for $28.8 \%$ of all fungal reads, and was present in every infant sample, ranging from 0.02 to $97.1 \%$ abundance (in F4_12 and M1_06, respectively). In total, six Candida species were 
identified, including most notably C. albicans, C. metapsilosis, C. parapsilosis and C. tropicalis, all of which are human-associated and fungal pathobionts [47]. In the case of the other two species, namely C. natalensis and C. railenensis, both were discounted from further analyses based on their inability to survive above $30^{\circ} \mathrm{C}[35]$.

Of the four human-associated Candida, at least one species was present in every infant sample with $79 \%$ of the samples containing two or more species with C. albicans and C. parapsilosis found in 17/23 (74\%) samples (Figure 2). Overall, C. parapsilosis was the most abundant (mean of 13.3\%) and prevalent $(90 \%)$. Only three infants did not carry C. parapsilosis at all timepoints (Figure 2). C. albicans was the second most abundant (mean of $10.9 \%$ ) and prevalent species $(66 \%)$. In contrast, C. metapsilosis and C. tropicalis were less common, present in four and eight samples, respectively. Despite its low prevalence, $C$. metapsilosis was nevertheless the most abundant species $(70.1 \%)$ in the 6-month sample of infant M5 (Figure 2). This infant was unique as he was the only member of the cohort categorized as 'high risk', based on his gestational age at birth ( $<27$ weeks) and a birthweight of $<750 \mathrm{~g}$. Such 'high risk' infants routinely receive prophylactic antifungals while fitted with an indwelling central venous catheter to help reduce the risk of fungal infection, and fluconazole was the prophylactic antifungal administered to this infant (from day 7 to 36 ; see Table S1).

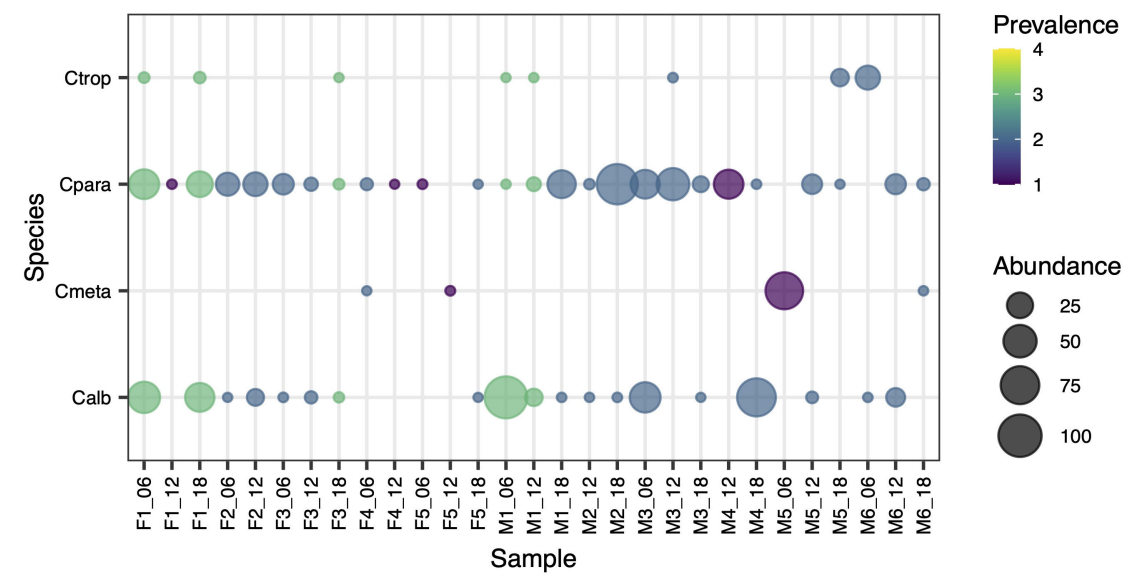

Figure 2. Bubble plot depicting the prevalence and relative abundance of opportunistic pathogenic Candida species in the preterm infant gut mycobiome (Calb, C. albicans; Cmeta, C. metapsilosis; Cpara, C. parapsilosis; Ctrop, C. tropicalis).

All infants were born premature and their gestational ages (GA) at birth ranged from 25 to 36 weeks (Table S1). When the longitudinal samples were re-ordered from earliest to latest GA, there were clear differences between the distribution and abundance of the four Candida species; most notably between C. albicans and C. parapsilosis (Figure 3). For the six infants born at $<31$ weeks, C. parapsilosis was the more prevalent, present in $14 / 16$ samples $(\sim 87 \%)$, compared to 9 samples $(\sim 56 \%)$ for C. albicans. Furthermore, C. parapsilosis was also more abundant than C. albicans in these infants $(12.6 \%$ versus $2.7 \%$, respectively). In contrast, for the five infants born at 31 weeks or later, while both species were present in a comparable number of samples (C. albicans, 10; C. parapsilosis, 12), C. albicans was more abundant (C. albicans, $21.0 \%$; C. parapsilosis, $14.1 \%$ ). Whereas the mean relative abundance of $C$. parapsilosis remained at a similar level between the two infant groups (GA: $<31$ weeks, $12.6 \%$; $\geq 31$ weeks, $14.1 \%$ ), it increased $\sim 8$-fold for $C$. albicans (GA: $<31$ weeks, $2.7 \%$; $\geq 31$ weeks, $21.0 \%$ ). 


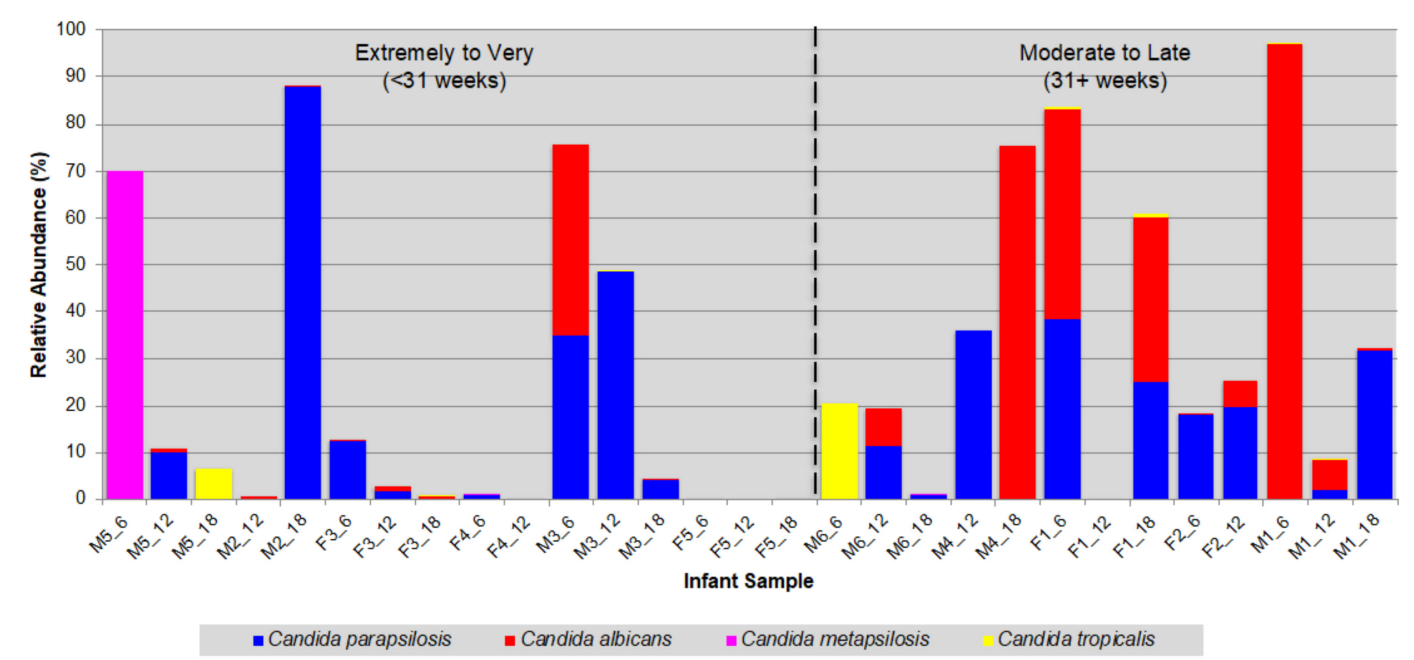

Figure 3. Bar graph of the prevalence and abundance of human-associated Candida species in the preterm infant gut mycobiome. Samples ordered according to infant gestational age at birth.

\subsection{Fungal Species Persistence}

Fungal persistence in serial samples is indicative of successful colonisation. To investigate this further, longitudinal samples from each individual infant were examined focusing on taxa identified to species level. A total of 23 species were identified of which nine appeared infant-specific (e.g., Aspergillus ruber, infant M1), with 14 re-occurring in two or more infants (e.g., Debaryomyces hansenii; infants F1, F3, M1, M3 and M6) (see Table S6).

By applying the criteria of survivability and growth at body temperature, 14 fungi were discounted as transients based on their inability to grow at $37^{\circ} \mathrm{C}$. The remaining nine species were further subdivided into three groups according to whether they were foodborne, of environmental origin or human-associated of which the latter group contained the majority of species. In addition to C. albicans, C. parapsilosis and C. tropicalis, which are considered human-associated species [47,48], this group also included Malassezia restricta, Meyerozyma guiliiermondi and Wickerhamomyces onychis (Table 1). The most persistent and re-occurring species able to grow at $37^{\circ} \mathrm{C}$ were C. albicans ( 7 infants), C. parapsilosis (11), D. hansenii (5), M. restricta (4) and S. cerevisiae (5).

Table 1. Persistent fungi. Species detected at two or more collection timepoints (i.e., at 6-, 12- and/or 18-months), with the ability to grow at body temperature.

\begin{tabular}{ccc}
\hline Species & Category & Infant \# \\
\hline Aspergillus ruber & Environmental & 1 \\
Candida albicans & Human-associated & 7 \\
Candida parapsilosis & Human-associated & 11 \\
Candida tropicalis $^{\text {Debaryomyces hansenii }}{ }^{1}$ & Human-associated & 2 \\
Malassezzia restricta & Foodborne & 5 \\
Meyerozyma guilliermondii & Human-associated & 4 \\
Saccharomyces cerevisiae $^{1}$ & Human-associated & 2 \\
Wickerhamomyces onychis $^{1}$ & Foodborne/Probiotic $^{2}$ & 5 \\
\hline
\end{tabular}

\# Number of infants carrying the species; ${ }^{1}$ Growth at $37^{\circ} \mathrm{C}$ is strain variable; ${ }^{2}$ Saccharomyces boulardii, a probiotic strain of S. cerevisiae. 


\subsection{Fungal Community Dynamics}

To determine how fungal diversity changed over time, the longitudinal samples were subdivided according to time of collection. The analysis showed that as the infants aged and their diet changed species diversity (as taxa/sample) increased, albeit gradually (see Table S2). This was irrespective of whether all 29 samples were included, or if the analysis was restricted to the infants for which three longitudinal samples were available. The increase in diversity was most marked in the seven infants from whom three samples were obtained, with the mean number of taxa per sample increasing from 14.4 at 6 months, to 20.6 at 18 months with the largest range in diversity being at 12 months (range: 5 to 37 taxa/sample) (Figure 4). Due to the small infant cohort size, limited number of samples, and difference in sequencing depth between samples, the observed increase in fungal diversity did not reach significance. However, when the analyses were repeated using only samples with $>10,000$ reads and the data rarefied, then the alpha diversity (using Shannon metrics) still increased as the infants aged (see Figure S3).

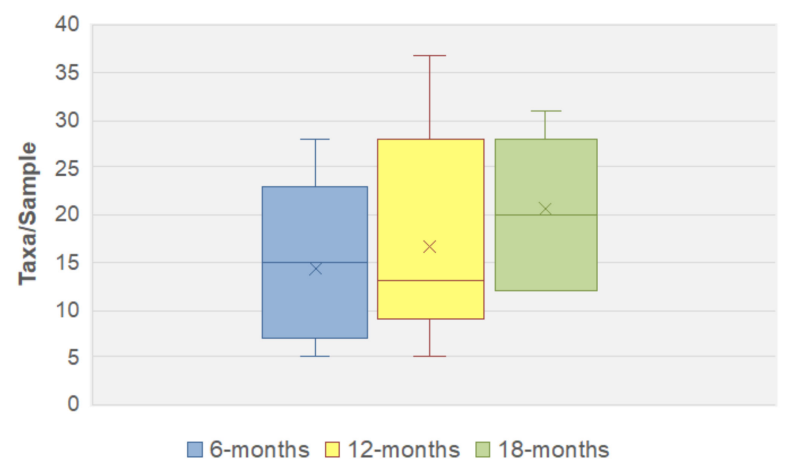

Figure 4. Fungal alpha-diversity depicted by a box-and-whisker plot showing number of taxa detected in each faecal sample for infants with 3 longitudinal samples. The horizontal line represents the median marker and ' $x$ ' represents the mean marker.

Two notable fungi which increased in prevalence over the 12-month study period were $D$. hansenii and S. cerevisiae. While both species were present at all three timepoints, the majority of infant samples in which they were detected, either individually or together, were at the 12 and 18-month timepoints (D. hansenii, 11/14; S. cerevisiae, 12/15) (Figure 5 and Table S7). The lowest abundance, of either species, was at 6 months whereas the highest abundance of either species was at 12 months. Indeed, S. cerevisiae was the dominant species in three of the 12-month samples, while D. hansenii accounted for almost all (99.4\%) ITS1 reads in F1_12 (Figure 5).

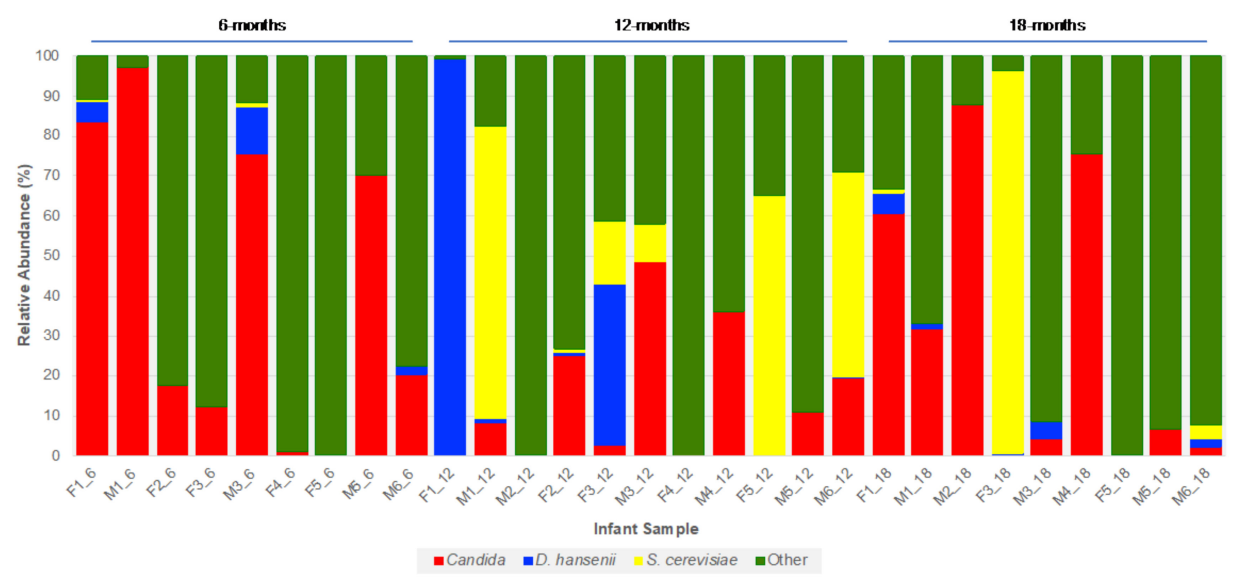

Figure 5. Bar chart depicting the prevalence and abundance of D. hansenii and S. cerevisiae in the preterm infant gut at between 6- and 18-months of age. 


\section{Discussion}

Preterm infants, with their immature immune system and an intestinal epithelial barrier of increased permeability, represent a highly vulnerable paediatric patient group. A shortened gestational period, disrupted in utero development, possible delivery by Caesarean section and significant antibiotic treatments all contribute to the bacterial composition of the preterm gut being distinct, typically characterised by reduced diversity and dominated by facultative anaerobes [22,23]. However, compared to the well-characterised bacterial community, very little is known about the mycobiome of the premature infant gut. Furthermore, while bacterial composition has been shown to differ during the first few weeks of life based on gestational maturity at birth [49], little is known about how gestational age affects and possibly shapes the enteric mycobiota of these infants.

To begin addressing this shortfall, we developed a workflow and protocols to characterise the fungal communities present in a cohort of infants, born with differing degrees of prematurity, and mapped how they develop during the first 18 months of life. Our findings reveal that the fungal communities within the preterm infant GI tract exhibit considerable diversity, were infant-specific, and characterised by the dominance of a single taxon and the presence of pathobiont Candida species. The makeup of the mycobiome is also influenced by GA and early life environmental factors.

Over 100 different fungi were identified with the majority classified to either the genus or species level. Despite this considerable diversity, many of the infant faecal samples contained a relatively low number of taxa, and were often dominated by a single taxon, typically a yeast from the genera Candida, Debaryomyces or Saccharomyces. (e.g., C. albicans, C. parapsilosis, D. hansenii or S. cerevisiae), with Candida colonisation influenced by the gestational age of the neonate at birth. Many of the fungi were infant-specific, often present in very low abundance, found at only one timepoint over the course of the 12-month study period and were not detected in the DNA extraction control. For those taxa for which a species identity could be determined $(\sim 70 \%)$, many were environmental fungi based on the fact they are commonly found in soil, the air, and associated with plants either as pathogens or saprophytes. Many of these fungi are incapable of growth at $37^{\circ} \mathrm{C}$ making their presence in the infant samples incidental, due to either ingestion (as foodborne contaminants) or through spore inhalation and subsequent ingestion. This is perhaps best exemplified by the spore-forming species Davidiella tassiana (anamorph-Cladosporium herbarum) and Baeospora myosura repeatedly detected in our preterm infant cohort, although often at low abundance. Their inability to grow at human body temperature means that their presence is most likely incidental due to spore ingestion (e.g., as foodborne contaminants).

At a phylum level, the preterm infant gut mycobiome comprises exclusively of fungi from the Ascomycota and Basidiomycota phyla, with Ascomycota dominating at all three timepoints over the 12 months. This dominance was largely due to the presence of the genera Candida, Davidiella, Debaryomyces, Penicillium and Saccharomyces, which collectively accounted for over $50 \%$ of all fungal reads. The dominance of Ascomycota and Basidiomycota in the healthy human GI tract, of both infants and adults, has been reported previously in several studies, with these fungi also being dominant members of the skin, vaginal and oral cavity mycobiomes [10,33,50-53]. Collectively, this suggests members of these phyla are well-suited for persisting within and on the human host. In addition, the mammalian GI tract appears to be the primary ecological niche for a number of human-associated Candida including most notably C. albicans, a species rarely found in the environment $[48,54]$.

Candida accounted for over a quarter of all the fungal reads and it was the most prevalent genus, being detected in every infant sample. This is in line with Candida species and C. albicans in particular being frequently identified both in the infant and adult gut $[33,34,53,55,56]$, as well as in other body sites, including the oral cavity and vagina $[50,51,57]$. Six Candida species were identified in the preterm infant cohort, including C. albicans, C. metapsilosis, C. parapsilosis and C. tropicalis, all of which are human-associated and fungal pathobionts [47]. Indeed, at least one of these four Candida species was detected in every infant sample with the majority containing at least two of the species, most commonly C. albicans and C. parapsilosis. This is consistent with preterm infants having higher Candida colonisation rates compared to their full-term counterparts [32]. Although infrequently detected, 
C. metapsilosis was most notable for being the dominant Candida pathobiont in the most premature infant (25 weeks), which uniquely received the prophylactic antifungal fluconazole in the first month of life. Our study also revealed that Candida colonisation persists well beyond the first few weeks after birth, when many preterm infants, especially those born $<28$ weeks, remain hospitalised and highly susceptible to nosocomial bacterial or fungal infection. Indeed, in two of the preterm infants, Candida are the dominant fungi at 18 months after birth (Infant M2, C. parapsilosis; M4, C. albicans).

Although C. albicans is a common commensal of both the human GI tract and oral cavity [58] and is a predominant member of the vaginal mycobiome [59], C. parapsilosis was the most prevalent Candida species in the preterm infant cohort. Of the 11 preterm infants studied, eight carried C. parapsilosis at all timepoints over the 12 months. It was also more abundant in those infants born at $<31$ weeks (i.e., very/extremely preterm), whereas $C$. albicans abundance was generally higher in infants born at $\geq 31$ weeks (i.e., moderately preterm to near term).

To date, little data is available relating to the composition of the enteric mycobiome of infants born full term. However, in a recent longitudinal study, where the anal, oral and skin mycobiomes of a cohort of term infants were characterized over the first month of life, C. parapsilosis along with C. albicans were both found to be prevalent in the anal mycobiome of these infants, present in $95 \%$ and $85 \%$ of samples, respectively [33]. This previous study also found C. tropicalis to be highly prevalent in these infants (present in $>90 \%$ anal samples), which is in marked contrast to the results from our study, where this species was detected in just over a quarter of infant samples. A possible explanation for this marked difference could be the fact the authors found C. tropicalis, as well as C. albicans, to be present in every maternal vaginal mycobiome [33], and thus its prevalence in these infants could reflect the fact they were all born to term. This may also account for why C. albicans, again present in every pregnant mother, was more prevalent in the term-born infants compared to the preterm infants in our study ( $85 \%$ and $66 \%$, respectively) [33].

Whilst C. parapsilosis is often detected in the human gut $[10,33,60]$, it is primarily a skin commensal [61] and is often present in the hospital environment, and in particular in the neonatal intensive care unit (NICU). C. parapsilosis is, therefore, considered a significant human pathogen, particularly of preterm neonates. Multiple risk factors including prolonged hospitalisation, very low birth weight $(<1500 \mathrm{~g})$, parenteral nutrition and the use of indwelling intravenous catheters, hand carriage and indirect (horizontal) transmission contribute to the vulnerability of preterm infants to C. parapsilosis infection $[29,62,63]$. All but one of our preterm cohort were born with either veryor extremely low birth weight, and required lengthy hospitalisation after birth (mean hospital stay: 49 days). The prevalence of $C$. parapsilosis in these infants may therefore result from hospital acquisition rather than vertical transfer from mother to offspring, either before or during delivery. Considering that all preterm infants endure disrupted fetal development, with the final weeks/months of development occurring ex utero and with little exposure to the maternal mycobiota, this may account for why C. albicans, a key member of the vaginal mycobiome [59], was less abundant than C. parapsilosis in these infants. However, in the absence of accompanying maternal mycobiota data, we cannot discount the possibility that these findings were due to or influenced by the composition of the maternal mycobiome.

Based on the growth requirements of individual fungi, we were able to discriminate between transient versus persistent colonisers of the preterm infant gut. On this basis, only nine species possessed the ability to grow at $37^{\circ} \mathrm{C}$, representing potential stable colonisers of which C. albicans and C. parapsilosis occurred the most, and are often found in the adult human gut $[33,34,53,55,56]$.

Three other notable persistent yeast species in the preterm infants were $D$. hansenii, $M$. restricta and S. cerevisiae. D. hansenii is a ubiquitous osmotolerant fungus, highly prevalent in dairy products and often used in the food industry as a cheese yeast $[64,65]$. D. hansenii has also been isolated from the skin and breast milk, and is a dominant member of the infant gut mycobiota during the breast-feeding period [10,65-67]. It has also been cultured from human faeces and some strains are reported to grow at $37^{\circ} \mathrm{C}$ [64]. Collectively, this would suggest $D$. hansenii or at least certain strains of the species have the capacity to survive and colonise the preterm infant GI tract. 
M. restricta is a lipophilic basidiomycete and, like other members of the genus, is considered to be one of the principle fungal constituents of the skin microbiota of infants and adults $[52,68,69]$. Thus, as a prevalent skin commensal, $M$. restricta acquisition can occur by either vertical transmission (mother to offspring) or horizontal transfer (healthcare worker to offspring). M. restricta has also been detected in human breast milk as well as the GI tract $[10,53,60,66,67,70]$. Whilst its presence in breast milk may be due to possible transfer from surrounding maternal skin, the fact it has been detected repeatedly in the human gut would indicate it can also colonise the GI tract $[10,53,60,70]$. This is supported by our finding that $M$. restricta is prevalent and persistent in four of the preterm infants.

S. cerevisiae persisted in almost half of the infant cohort, and increased in abundance over time in two of the infants, indicative of successful colonisation. The findings that $S$. cerevisiae is frequently detected in the adult human GI tract $[10,48,71]$ and that some strains grow well at $37^{\circ} \mathrm{C}[66,72]$ is consistent with $S$. cerevisiae being a stable coloniser of the human GI tract. Countering this claim for S. cerevisiae being a human GI tract commensal is the recent finding that $S$. cerevisiae could not be detected in stool samples of four healthy Western adults fed a S. cerevisiae-free diet [73]. This finding and study suggests it is a transient coloniser of the human GI tract, with its presence being dependent upon sustained consumption of food containing S. cerevisiae (e.g., bread) [48]. Further studies are required to establish the true nature of the relationship between $S$. cerevisiae and the human mycobiome.

Overall, the diversity of the preterm infant gut mycobiome is low, with community composition differing between infants. Similar findings have been reported for full term infants $[10,33]$ suggesting that in early life the infant gut is populated by a relatively small number of fungal species, irrespective of whether the infant is born premature or to term. Through longitudinal sampling, we observed that as the infants aged, so the diversity and species richness of their respective gut mycobiomes also increased, albeit gradually as noted in full term infants [10]. Diet is likely to be a major factor and driver of this increased diversity. Between 6 and 9 months of age, most babies typically begin weaning (or complimentary feeding), a time when they are first introduced to solid foods. At this point in development, and over the coming months, not only does their diet increase in complexity, from purely milk-based, but these infants are also exposed to a variety of different foodborne microbes including fungi. The persistence of $D$. hansenii and S. cerevisiae that are both closely associated with food in our cohort highlight the importance of the post-weaning infant diet and the role it can play in fungal acquisition and in shaping the early life preterm infant gut mycobiome.

\section{Conclusions}

This study describes the development of a protocol and pipeline workflow for the characterisation of the human faecal mycobiome and its application to provide the first detailed insight into the fungal communities present within the preterm infant gut in early life. Our findings show how colonisation of the preterm infant gut is influenced by gestational age, and how age and diet impact on the subsequent development and diversity of the gut mycobiome. A characteristic feature of the preterm infant gut mycobiome is the prominence of the pathobiont C. parapsilosis, a prevalent hospital-associated fungal pathobiont. This study paves the way for further investigations of the developing enteric mycobiome and the importance and impact of gestational maturity at birth on community composition, as well as early antibiotic/antifungal prophylaxis in establishing both a healthy mycobiome and microbiome. Furthermore, while probiotic supplementation in preterm infants has been shown to have a positive impact, leading to a Bifidobacterium-dominated gut with a lower abundance of bacterial pathobionts, no such study has yet been carried out to investigate the effect of such treatment on the gut mycobiota of these infants. 
Supplementary Materials: The following are available online at http://www.mdpi.com/2309-608X/6/4/273/s1, Figure S1: Workflow diagram of extraction protocol (left hand panel) and bioinformatic analysis pipeline (right hand panel), Figure S2: Fungal abundance over time (at phylum level), Figure S3: Fungal alpha diversity (Shannon diversity index values for samples with $>10,000$ reads sequencing depth and data rarefication), Datasheet S1: Fungal taxa relative abundance table, Table S1: Infant metadata, Table S2: Fungal taxa/infant sample, Table S3: Fungal taxa identified to species level, Table S4: Genus abundance and prevalence, Table S5: Most abundant taxa, Table S6: Persistent fungal species, Table S7: Relative abundance values of D. hansenii and S. cerevisiae at 6-, 12and 18-months.

Author Contributions: Conceptualization, S.R.C., L.J.H. and S.A.J.; methodology, S.A.J., S.P., A.T., R.A. and D.B.; software, A.T. and R.A.; validation, S.A.J., A.T. and R.A.; formal analysis, A.T. and S.A.J.; resources, S.P. and P.C.; data curation, A.T.; writing-original draft preparation, S.A.J.; writing-review and editing, S.R.C., L.J.H., P.C. and S.A.J.; supervision, S.R.C.; project administration, S.R.C.; funding acquisition, S.R.C. and L.J.H. All authors have read and agreed to the published version of the manuscript.

Funding: This research was supported in part by Biotechnology and Biological Sciences Research Council (BBSRC) Institute Strategic Programme grants awarded to the Gut Health and Food Safety (BB/J004529/1; S.R.C.) and Gut Microbes and Health (BB/R012490/1, (BBS/E/F/000PR10353, BBS/E/F/000PR10355 and BBS/E/F/000PR10356; L.J.H. and S.R.C.) research programmes. Additional support was provided by a Wellcome Trust Investigator Award (100974/C/13/Z, L.J.H.).

Acknowledgments: We thank all clinical nurses at Norfolk and Norwich University Hospital (NNUH) for collecting stool samples. We also acknowledge the support of research nurses Karen Few, Kathryn McColl, Hayley Aylmer and Zoe McClure in obtaining informed consent and collecting samples.

Conflicts of Interest: The authors declare no conflict of interest. The funders had no role in the design of the study; in the collection, analyses, or interpretation of data; in the writing of the manuscript, or in the decision to publish the results.

\section{References}

1. Qin, J.J.; Li, R.Q.; Raes, J.; Arumugam, M.; Burgdorf, K.S.; Manichanh, C.; Nielsen, T.; Pons, N.; Levenez, F.; Yamada, T.; et al. A human gut microbial gene catalogue established by metagenomic sequencing. Nature 2010, 464, 59-65. [CrossRef] [PubMed]

2. Huttenhower, C.; Gevers, D.; Knight, R.; Abubucker, S.; Badger, J.H.; Chinwalla, A.T.; Creasy, H.H.; Earl, A.M.; FitzGerald, M.G.; Fulton, R.S.; et al. Structure, function and diversity of the healthy human microbiome. Nature 2012, 486, 207-214. [CrossRef]

3. Shreiner, A.B.; Kao, J.Y.; Young, V.B. The gut microbiome in health and in disease. Curr. Opin. Gastroenterol. 2015, 31, 69-75. [CrossRef] [PubMed]

4. Groer, M.W.; Luciano, A.A.; Dishaw, L.J.; Ashmeade, T.L.; Miller, E.; Gilbert, J.A. Development of the preterm infant gut microbiome: A research priority. Microbiome 2014, 2. [CrossRef] [PubMed]

5. Mueller, N.T.; Whyatt, R.; Hoepner, L.; Oberfield, S.; Dominguez-Bello, M.G.; Widen, E.M.; Hassoun, A.; Perera, F.; Rundle, A. Prenatal exposure to antibiotics, cesarean section and risk of childhood obesity. Int. J. Obes. 2015, 39, 665-670. [CrossRef]

6. Fujimura, K.E.; Sitarik, A.R.; Haystad, S.; Lin, D.L.; Levan, S.; Fadrosh, D.; Panzer, A.R.; LaMere, B.; Rackaityte, E.; Lukacs, N.W.; et al. Neonatal gut microbiota associates with childhood multisensitized atopy and T cell differentiation. Nat. Med. 2016, 22, 1187-1191. [CrossRef]

7. Tamburini, S.; Shen, N.; Wu, H.C.; Clemente, J.C. The microbiome in early life: Implications for health outcomes. Nat. Med. 2016, 22, 713-722. [CrossRef]

8. Pascal, M.; Perez-Gordo, M.; Caballero, T.; Escribese, M.M.; Longo, M.N.L.; Luengo, O.; Manso, L.; Matheu, V.; Seoane, E.; Zamorano, M.; et al. Microbiome and Allergic Diseases. Front. Immunol. 2018, 9. [CrossRef] [PubMed]

9. Huffnagle, G.B.; Noverr, M.C. The emerging world of the fungal microbiome. Trends Microbiol. 2013, 21, 334-341. [CrossRef] [PubMed]

10. Schei, K.; Avershina, E.; Oien, T.; Rudi, K.; Follestad, T.; Salamati, S.; Odegard, R.A. Early gut mycobiota and mother-offspring transfer. Microbiome 2017, 5. [CrossRef]

11. Underhill, D.M.; Lliev, L.D. The mycobiota: Interactions between commensal fungi and the host immune system. Nat. Rev. Immunol. 2014, 14, 405-416. [CrossRef]

12. Szajewska, H.; Mrukowicz, J. Meta-analysis: Non-pathogenic yeast Saccharomyces boulardii in the prevention of antibiotic-associated diarrhoea. Aliment. Pharmacol. Ther. 2005, 22, 365-372. [CrossRef] 
13. Zanello, G.; Meurens, F.; Berri, M.; Salmon, H. Saccharomyces boulardii effects on gastrointestinal diseases. Curr. Issues Mol. Biol. 2009, 11, 47-58. [PubMed]

14. Hatoum, R.; Labrie, S.; Fliss, I. Antimicrobial and probiotic properties of yeasts: From fundamental to novel applications. Front. Microbiol. 2012, 3. [CrossRef]

15. Feizizadeh, S.; Salehi-Abargouei, A.; Akbari, V. Efficacy and Safety of Saccharomyces boulardii for Acute Diarrhea. Pediatrics 2014, 134, E176-E191. [CrossRef]

16. Chehoud, C.; Judge, C.; Hoffmann, C.; Grunberg, S.; Bittinger, K.; Baldassano, R.N.; Lewis, J.D.; Bushman, F.D.; Wu, G.D. Fungal Signature in the Gut Microbiota of Pediatric Patients With Inflammatory Bowel Disease. Inflamm. Bowel Dis. 2015, 21, 1948-1956. [CrossRef]

17. Sokol, H.; Leducq, V.; Aschard, H.; Pham, H.P.; Jegou, S.; Landman, C.; Cohen, D.; Liguori, G.; Bourrier, A.; Nion-Larmurier, I.; et al. Fungal Microbiota Dysbiosis IBD. Gut 2017, 66, 1039-1048. [CrossRef]

18. Luan, C.G.; Xie, L.L.; Yang, X.; Miao, H.F.; Lv, N.; Zhang, R.F.; Xiao, X.; Hu, Y.F.; Liu, Y.L.; Wu, N.; et al. Dysbiosis of Fungal Microbiota in the Intestinal Mucosa of Patients with Colorectal Adenomas. Sci. Rep. 2015, 5. [CrossRef]

19. Rodriguez, M.M.; Perez, D.; Chaves, F.J.; Esteve, E.; Marin-Garcia, P.; Xifra, G.; Vendrell, J.; Jove, M.; Pamplona, R.; Ricart, W.; et al. Obesity changes the human gut mycobiome. Sci. Rep. 2015, 5. [CrossRef]

20. Palmer, C.; Bik, E.M.; DiGiulio, D.B.; Relman, D.A.; Brown, P.O. Development of the human infant intestinal microbiota. PloS Biol. 2007, 5, 1556-1573. [CrossRef]

21. Turnbaugh, P.J.; Hamady, M.; Yatsunenko, T.; Cantarel, B.L.; Duncan, A.; Ley, R.E.; Sogin, M.L.; Jones, W.J.; Roe, B.A.; Affourtit, J.P.; et al. A core gut microbiome in obese and lean twins. Nature 2009, 457, 480-484. [CrossRef]

22. Henderickx, J.G.E.; Zwittink, R.D.; van Lingen, R.A.; Knol, J.; Belzer, C. The Preterm Gut Microbiota: An Inconspicuous Challenge in Nutritional Neonatal Care. Front. Cell. Infect. Microbiol. 2019, 9. [CrossRef] [PubMed]

23. Tirone, C.; Pezza, L.; Paladini, A.; Tana, M.; Aurilia, C.; Lio, A.; D’Ippolito, S.; Tersigni, C.; Posteraro, B.; Sanguinetti, M.; et al. Gut and Lung Microbiota in Preterm Infants: Immunological Modulation and Implication in Neonatal Outcomes. Front. Immunol. 2019, 10. [CrossRef]

24. Rice, L.B. Federal funding for the study of antimicrobial resistance in nosocomial pathogens: No ESKAPE. J. Infect. Dis. 2008, 197, 1079-1081. [CrossRef]

25. Neu, J.; Walker, W.A. Medical Progress: Necrotizing Enterocolitis. N. Engl. J. Med. 2011, 364, $255-264$. [CrossRef]

26. Pammi, M.; Weisman, L.E. Late-onset sepsis in preterm infants: Update on strategies for therapy and prevention. Expert Rev. Anti-Infect. Ther. 2015, 13, 487-504. [CrossRef]

27. Shulhan, J.; Dicken, B.; Hartling, L.; Larsen, B.M.K. Current Knowledge of Necrotizing Enterocolitis in Preterm Infants and the Impact of Different Types of Enteral Nutrition Products. Adv. Nutr. 2017, 8, 80-91. [CrossRef]

28. Saiman, L.; Ludington, E.; Pfaller, M.; Rangel-Frausto, S.; Wiblin, R.T.; Dawson, J.; Blumberg, H.M.; Patterson, J.E.; Rinaldi, M.; Edwards, J.E.; et al. Risk factors for candidemia in neonatal intensive care unit patients. Pediatr. Infect. Dis. J. 2000, 19, 319-324. [CrossRef] [PubMed]

29. Lupetti, A.; Tavanti, A.; Davini, P.; Ghelardi, E.; Corsini, V.; Merusi, I.; Boldrini, A.; Campa, M.; Senesi, S. Horizontal transmission of Candida parapsilosis candidemia in a neonatal intensive care unit. J. Clin. Microbiol. 2002, 40, 2363-2369. [CrossRef]

30. Bliss, J.M.; Basavegowda, K.P.; Watson, W.J.; Sheikh, A.U.; Ryan, R.M. Vertical and horizontal transmission of Candida albicans in very low birth weight infants using DNA fingerprinting techniques. Pediatr. Infect. Dis. J. 2008, 27, 231-235. [CrossRef] [PubMed]

31. Sabino, R.; Sampaio, P.; Carneiro, C.; Rosado, L.; Pais, C. Isolates from hospital environments are the most virulent of the Candida parapsilosis complex. BMC Microbiol. 2011, 11. [CrossRef] [PubMed]

32. Pammi, M.; Holland, L.; Butler, G.; Gacser, A.; Bliss, J.M. Candida parapsilosis Is a Significant Neonatal Pathogen: A Systematic Review and Meta-analysis. Pediatr. Infect. Dis. J. 2013, 32, E206-E216. [CrossRef]

33. Ward, T.L.; Dominguez-Bello, M.G.; Heisel, T.; Al-Ghalith, G.; Knights, D.; Gale, C.A. Development of the Human Mycobiome over the First Month of Life and across Body Sites. mSystems 2018, 3. [CrossRef]

34. Heisel, T.; Nyaribo, L.; Sadowsky, M.J.; Gale, C.A. Breastmilk and NICU surfaces are potential sources of fungi for infant mycobiomes. Fungal Genet Biol. 2019, 128, 29-35. [CrossRef] 
35. Alcon-Giner, C.; Dalby, M.J.; Caim, S.; Ketskemety, J.; Shaw, A.; Sim, K.; Lawson, M.A.E.; Kiu, R.; Leclaire, C.; Chalkien, L.; et al. Microbiota Supplementation with Bifidobacterium and Lactobacillus Modifies the Preterm Infant Gut Microbiota and Metabolome: An Observational Study. Cell Rep. Med. 2020, 1. [CrossRef]

36. White, T.J.; Bruns, T.D.; Lee, S.L.; Taylor, J.W. Amplification and Direct Sequencing of Fungal Ribosomal RNA Genes for Phylogenetics. In PCR Protocols: A Guide to Methods and Applications; Innis, M.A., Gelfand, D.H., Sninsky, J.J., Eds.; Academic Press: San Diego, CA, USA, 1990; pp. 315-322.

37. Gardes, M.; Bruns, T.D. Its Primers with Enhanced Specificity for Basidiomycetes-Application to the Identification of Mycorrhizae and Rusts. Mol. Ecol. 1993, 2, 113-118. [CrossRef]

38. Edgar, R.C. Search and clustering orders of magnitude faster than BLAST. Bioinformatics 2010, 26, $2460-2461$. [CrossRef]

39. Bolyen, E.; Rideout, J.R.; Dillon, M.R.; Bokulich, N.A.; Abnet, C.C.; Al-Ghalith, G.A.; Alexander, H.; Alm, E.J.; Arumugam, M.; Asnicar, F.; et al. Reproducible, interactive, scalable and extensible microbiome data science using QIIME 2. Nat. Biotechnol. 2019, 37, 1091. [CrossRef] [PubMed]

40. Chen, S.F.; Zhou, Y.Q.; Chen, Y.R.; Gu, J. fastp: An ultra-fast all-in-one FASTQ preprocessor. Bioinformatics 2018, 34, 884-890. [CrossRef] [PubMed]

41. Edgar, R.C. UNOISE2: Improved error-correction for Illumina $16 \mathrm{~S}$ and ITS amplicon sequencing. bioRxiv 2016, 081257. [CrossRef]

42. Nilsson, R.H.; Larsson, K.H.; Taylor, A.F.S.; Bengtsson-Palme, J.; Jeppesen, T.S.; Schigel, D.; Kennedy, P.; Picard, K.; Glockner, F.O.; Tedersoo, L.; et al. The UNITE database for molecular identification of fungi: Handling dark taxa and parallel taxonomic classifications. Nucleic Acids Res. 2019, 47, D259-D264. [CrossRef] [PubMed]

43. Katoh, K.; Misawa, K.; Kuma, K.; Miyata, T. MAFFT: A novel method for rapid multiple sequence alignment based on fast Fourier transform. Nucleic Acids Res. 2002, 30, 3059-3066. [CrossRef] [PubMed]

44. Price, M.N.; Dehal, P.S.; Arkin, A.P. FastTree 2-Approximately Maximum-Likelihood Trees for Large Alignments. PLoS ONE 2010, 5. [CrossRef] [PubMed]

45. R Development Core Team. R: A Language and Environment for Statistical Computing; R Foundation for Statistical Computing: Vienna, Austria, 2020; Available online: https://www.R-project.org/ (accessed on 10 October 2020).

46. McMurdie, P.J.; Holmes, S. Phyloseq: An R Package for Reproducible Interactive Analysis and Graphics of Microbiome Census Data. PLoS ONE 2013, 8, e61217. [CrossRef]

47. Lachance, M.A.; Boekhout, T.; Scorzetti, G.; Fell, J.W.; Kurtzman, C.P. Candida Berkhout (1923). In The Yeasts: A Taxonomic Study; Kurtzman, C.P., Fell, J.W., Boekhout, T., Eds.; Elsevier: Amsterdam, The Netherlands, 2011; pp. 987-1278.

48. Hallen-Adams, H.E.; Suhr, M.J. Fungi in the healthy human gastrointestinal tract. Virulence 2017, 8, 352-358. [CrossRef]

49. Chernikova, D.A.; Madan, J.C.; Housman, M.L.; Zain-ul-Abideen, M.; Lundgren, S.N.; Morrison, H.G.; Sogin, M.L.; Williams, S.M.; Moore, J.H.; Karagas, M.R.; et al. The premature infant gut microbiome during the first 6 weeks of life differs based on gestational maturity at birth. Pediatr. Res. 2018, 84, 71-79. [CrossRef]

50. Ghannoum, M.A.; Jurevic, R.J.; Mukherjee, P.K.; Cui, F.; Sikaroodi, M.; Naqvi, A.; Gillevet, P.M. Characterization of the Oral Fungal Microbiome (Mycobiome) in Healthy Individuals. PLoS Pathog. 2010, 6, e1000713. [CrossRef]

51. Drell, T.; Lillsaar, T.; Tummeleht, L.; Simm, J.; Aaspollu, A.; Vain, E.; Saarma, I.; Salumets, A.; Donders, G.G.G.; Metsis, M. Characterization of the Vaginal Micro- and Mycobiome in Asymptomatic Reproductive-Age Estonian Women. PLoS ONE 2013, 8, e54379. [CrossRef]

52. Findley, K.; Oh, J.; Yang, J.; Conlan, S.; Deming, C.; Meyer, J.A.; Schoenfeld, D.; Nomicos, E.; Park, M.; Kong, H.H.; et al. Topographic diversity of fungal and bacterial communities in human skin. Nature 2013, 498, 367. [CrossRef]

53. Nash, A.K.; Auchtung, T.A.; Wong, M.C.; Smith, D.P.; Gesell, J.R.; Ross, M.C.; Stewart, C.J.; Metcalf, G.A.; Muzny, D.M.; Gibbs, R.A.; et al. The gut mycobiome of the Human Microbiome Project healthy cohort. Microbiome 2017, 5, 1-13. [CrossRef]

54. Bensasson, D.; Dicks, J.; Ludwig, J.M.; Bond, C.J.; Elliston, A.; Roberts, I.N.; James, S.A. Diverse Lineages of Candida albicans Live on Old Oaks. Genetics 2019, 211, 277-288. [CrossRef] 
55. Hoffmann, C.; Dollive, S.; Grunberg, S.; Chen, J.; Li, H.Z.; Wu, G.D.; Lewis, J.D.; Bushman, F.D. Archaea and Fungi of the Human Gut Microbiome: Correlations with Diet and Bacterial Residents. PLoS ONE 2013, 8 , e66019. [CrossRef]

56. Hallen-Adams, H.E.; Kachman, S.D.; Kim, J.; Legge, R.M.; Martinez, I. Fungi inhabiting the healthy human gastrointestinal tract: A diverse and dynamic community. Fungal Ecol. 2015, 15, 9-17. [CrossRef]

57. Bradford, L.L.; Ravel, J. The vaginal mycobiome: A contemporary perspective on fungi in women's health and diseases. Virulence 2017, 8, 342-351. [CrossRef] [PubMed]

58. Moran, G.; Coleman, D.; Sullivan, D. An introduction to the medically important Candida species. In Candida and Candidiasis; Calderone, R.A., Clancy, C.J., Eds.; ASM Press: Washington, DC, USA, 2012; pp. 11-25.

59. Sobel, J.D. Vulvovaginal candidosis. Lancet 2007, 369, 1961-1971. [CrossRef]

60. Strati, F.; Di Paola, M.; Stefanini, I.; Albanese, D.; Rizzetto, L.; Lionetti, P.; Calabro, A.; Jousson, O.; Donati, C.; Cavalieri, D.; et al. Age and Gender Affect the Composition of Fungal Population of the Human Gastrointestinal Tract. Front. Microbiol. 2016, 7, 1227. [CrossRef]

61. Gacser, A. Adhesins in Candida parapsilosis: Understudied players in virulence. Virulence 2016, 7, 65-67. [CrossRef]

62. Van Asbeck, E.C.; Huang, Y.C.; Markham, A.N.; Clemons, K.V.; Stevens, D.A. Candida parapsilosis fungemia in neonates: Genotyping results suggest healthcare workers hands as source, and review of published studies. Mycopathologia 2007, 164, 287-293. [CrossRef]

63. Reiss, E.; Lasker, B.A.; Lott, T.J.; Bendel, C.M.; Kaufman, D.A.; Hazen, K.C.; Wade, K.C.; McGowan, K.L.; Lockhart, S.R. Genotyping of Candida parapsilosis from three neonatal intensive care units (NICUs) using a panel of five multilocus microsatellite markers: Broad genetic diversity and a cluster of related strains in one NICU. Infect. Genet. Evol. 2012, 12, 1654-1660. [CrossRef] [PubMed]

64. Breuer, U.; Harms, H. Debaryomyces hansenii-An extremophilic yeast with biotech nological potential. Yeast 2006, 23, 415-437. [CrossRef]

65. Suzuki, M.; Prasad, G.S.; Kurtzman, C.P. Debaryomyces Lodder \& Kreger-van Rij (1952). In The Yeasts: A Taxonomic Study; Kurtzman, C.P., Fell, J.W., Boekhout, T., Eds.; Elsevier: Amsterdam, The Netherlands, 2011; pp. 361-372.

66. Boix-Amoros, A.; Martinez-Costa, C.; Querol, A.; Collado, M.C.; Mira, A. Multiple Approaches Detect the Presence of Fungi in Human Breastmilk Samples from Healthy Mothers. Sci. Rep. 2018, 8, 1-13. [CrossRef]

67. Boix-Amoros, A.; Puente-Sanchez, F.; du Toit, E.; Linderborg, K.M.; Zhang, Y.M.; Yang, B.R.; Salminen, S.; Isolauri, E.; Tamames, J.; Mira, A.; et al. Mycobiome Profiles in Breast Milk from Healthy Women Depend on Mode of Delivery, Geographic Location, and Interaction with Bacteria. Appl. Environ. Microbiol. 2019, 85, 13. [CrossRef]

68. Gueho-Kellermann, E.; Batra, R.; Boekhout, T. Malassezia Baillon (1889). In The Yeasts: A Taxonomic Study; Kurtzman, C.P., Fell, J.W., Boekhout, T., Eds.; Elsevier: Amsterdam, The Netherlands, 2011; pp. 1807-1832.

69. Saunders, C.W.; Scheynius, A.; Heitman, J. Malassezia Fungi Are Specialized to Live on Skin and Associated with Dandruff, Eczema, and Other Skin Diseases. PLoS Pathog. 2012, 8, e1002701. [CrossRef] [PubMed]

70. Richard, M.L.; Lamas, B.; Liguori, G.; Hoffmann, T.W.; Sokol, H. Gut Fungal Microbiota: The Yin and Yang of Inflammatory Bowel Disease. Inflamm. Bowel Dis. 2015, 21, 656-665. [CrossRef]

71. David, L.A.; Maurice, C.F.; Carmody, R.N.; Gootenberg, D.B.; Button, J.E.; Wolfe, B.E.; Ling, A.V.; Devlin, A.S.; Varma, Y.; Fischbach, M.A.; et al. Diet rapidly and reproducibly alters the human gut microbiome. Nature 2014, 505, 559. [CrossRef] 
72. Vaughan-Martini, A.; Martini, A. Saccharomyces Meyen \& Reess (1870). In The Yeasts: A Taxonomic Study; Kurtzman, C.P., Fell, J.W., Boekhout, T., Eds.; Elsevier: Amsterdam, The Netherlands, 2011; pp. 733-746.

73. Auchtung, T.A.; Fofanova, T.Y.; Stewart, C.J.; Nash, A.K.; Wong, M.C.; Gesell, J.R.; Auchtung, J.M.; Ajami, N.J.; Petrosino, J.F. Investigating Colonization of the Healthy Adult Gastrointestinal Tract by Fungi. mSphere 2018, 3, e00092-18. [CrossRef] [PubMed]

Publisher's Note: MDPI stays neutral with regard to jurisdictional claims in published maps and institutional affiliations.

(C) 2020 by the authors. Licensee MDPI, Basel, Switzerland. This article is an open access article distributed under the terms and conditions of the Creative Commons Attribution (CC BY) license (http://creativecommons.org/licenses/by/4.0/). 